\title{
Kinesiology Tape does not Affect Serum Creatine Kinase Level and Quadriceps Activity during Recovery from Delayed-Onset Muscle Soreness
}

\author{
Naoko Aminaka (Corresponding author) \\ Department of Exercise and Sport Science \\ University of Wisconsin - La Crosse, La Crosse, WI USA \\ E-mail: naminaka@uwlax.edu \\ Tiffany Fohey \\ Department of Exercise and Sport Science \\ University of Wisconsin - La Crosse, La Crosse, WI USA \\ E-mail: fohey.tiff@uwlax.edu \\ Attila Kovacs \\ Department of Exercise and Sport Science \\ University of Wisconsin - La Crosse, La Crosse, WI USA \\ E-mail: akovacs@uwlax.edu \\ Roksana Zak \\ School of Health, Physical Education and Recreation \\ University of Nebraska Omaha, Omaha, NE USA \\ E-mail:rzak@unomaha.edu
}

Received: 19-12- 2016

doi:10.7575/aiac.ijkss.v.5n.1p.17
Accepted: 25-01- 2017

Published: 31-01- 2017

URL: http://dx.doi.org/10.7575/aiac.ijkss.v.5n.1p.17

\begin{abstract}
Background: Delayed-onset muscle soreness (DOMS) causes muscle damage and edema that can hinder performance and increase risks for secondary injuries. Kinesiology Tape (KT) may be an effective modality for aiding in recovery, however, no study has investigated the effects of KT on the physiological biomarkers such as serum creatine kinase (CK) level, concurrently with measures of performance and function, during recovery from DOMS. Objective: Investigate the effects of KT on serum CK level, electromyographic (EMG) activity of the quadriceps muscles, and performances of countermovement jump (CMJ) and triple single-leg hop for distance (HopD) during recovery from DOMS. Method: Fifty-eight healthy college-age participants were randomly assigned to KT $(\mathrm{n}=15)$, placebo $(\mathrm{n}=19)$, and control $(n=24)$ groups. Serum CK level and quadriceps EMG activity and performance during CMJ and HopD were collected at baseline, immediately after repetitive eccentric quadriceps exercise, 48 hours, and 72 hours post-exercise. The EMG recording of rectus femoris, vastus medialis, and vastus lateralis during the CMJ and HopD were normalized to the baseline maximum voluntary isometric contraction. Results: A significant main effect of time on the serum CK level, EMG activity, and performance $(\mathrm{p}<0.05)$ was observed. However, there were no group differences on the serum CK level, EMG activity, or performance ( $>>0.05)$. Conclusion: Taping interventions did not improve the serum CK level or muscle activity and performance during recovery from DOMS. Kinesiology tape may not be the first choice of method for enhancing recovery from DOMS in otherwise healthy individuals.
\end{abstract}

Keywords: therapeutic taping, muscle activity, creatine kinase, muscle soreness

\section{Introduction}

Delayed-onset muscle soreness (DOMS) is a phenomenon often presented with temporary damage and inability of a muscle to contract properly, occurring after a bout of repetitive eccentric contraction exercise (McHugh, Connolly, Eston, \& Gleim, 1999; Prasartwuth, Taylor, \& Gandevia, 2005). There may be swelling, pain, and tenderness associated with DOMS. DOMS typically occurs between 6 and 24 hours after repetitive eccentric contraction exercise, and peaks at 24-48 hours (Howell, Chleboum, \& Conaster, 1993). Signs and symptoms of DOMS can last for up to 72-96 hours, however, in severe cases they could last for 7 days (Curtis, Fallows, Morris, \& McMakin, 2010). DOMS causes relatively small damage to the muscle fibers, and with adequate amount of rest muscles may eventually hypertrophy in order to withstand the demand (Cheung, Hume, \& Maxwell, 2003). However, the symptoms associated with DOMS including inflammation, swelling, and pain can keep the athletes or other physically active individuals from performing at their optimum levels (Cheung et al., 2003). Since competitive and recreational athletes will usually try to work 
through these symptoms to avoid losing essential training time, the risks of secondary injuries to DOMS such as acute strains, hairline fractures, and rhabdomyolysis may increase (Baird, Graham, Baker, \& Bickerstaff, 2012; Page, 1995). Therefore, timely management of DOMS is imperative for safe participation in any physical activity.

One of the leading theories for the occurrence of DOMS is the mechanical theory, which states that eccentric contractions are the main culprit (Cheung et al., 2003; Tee, Bosch, \& Lambert, 2007). Damage to muscle fibers causes some proteins and electrolytes to spill out into the bloodstream. One of these spillage enzymes of particular importance is Creatine Kinase (CK). CK is an enzyme that plays a key role in cellular energetics, and is located near sites in the body where force generation by motor proteins, ion transport, and ATP processes take place, especially in the skeletal muscle (Wallimann, Wyss, Brdiczka, Nicolay, \& Eppenberger, 1992). CK catalyzes the reversible ATP-ADP hydrolysis reaction (Creatine + ATP $\rightleftarrows$ Phosphocreatine + ADP), and helps decipher muscle tissue damage at the cellular subunit level (Szasz, Gruber, \& Bernt, 1976). CK has been used as an indicator of DOMS in other research, with elevated levels seen during conditions that produce damage to skeletal muscles (Baird et al., 2012).

There are various therapeutic interventions to reduce tissue damage and inflammation associated with DOMS. One of the most functional modalities is Kinesiology Tape (KT). KT has become a popular choice of therapeutic modality in various clinical settings, due to its cost-effectiveness and its ability to allow functional range of motion during recovery (Bassett, Lingman, \& Ellis, 2010; Kase, Wallis, \& Kase, 2003; Thelen, Dauber, \& Stoneman, 2008). KT has many proposed mechanisms, including increasing proprioception, correcting muscle function by strengthening weakened muscles, improving circulation of blood and lymph by eliminating tissue fluid, and decreasing pain through neurological suppression (Fu et al., 2008; Ozmen et al., 2016; Williams, Whatman, Hume, \& Sheerin, 2012). Although research focuses more on KT and its effectiveness on pain reduction, limited data exists focusing on the effects of KT on lymph drainage in association to muscle eccentric overload. A few studies have shown some benefits of KT on edema reduction in injurious populations. KT is suggested to provide a mechanism for effectively facilitating lymph drainage by providing a light, directional stretch that massages superficial lymphatic vessels (Villeco, 2012). Taping technique, including the direction and location, may also play a role in determining how much massage is provided. Another study observed a significant swelling reduction with KT two days post operation for zygomatico-orbital fractures (Ristow et al., 2014). Furthermore, patients with breast cancer-related lymphedema demonstrated a swelling reduction with KT, along with more patient acceptance and compliance (Tsai, Hung, Yang, Huang, \& Tsauo, 2009). Although DOMS creates physiological changes such as swelling and muscle damage that are similar to changes associated with injurious/pathological conditions, the therapeutic effects of KT in the healthy population have been inconclusive (J. L. Drouin, McAlpine, Primak, \& Kissel, 2013; Duruturk, Pekyavas, Yilmaz, \& Karatas, 2016; Fu et al., 2008; Poon et al., 2015; Slupik, Dwornik, Bialoszewski, \& Zych, 2007). The evidence on the effects of KT on the biomarker functions such as CK is very limited (Hazar et al 2014), and further studies are warranted with a larger sample size and a control group.

The purpose of this study was to investigate the effects of KT on serum CK level, EMG activity, and performance of countermovement jump (CMJ) and triple single-hop test for distance (HopD) during the 72-hour recovery period from DOMS. We hypothesized that KT will result in decreased serum CK levels over the 72 hour recovery period, and a higher EMG amplitude (Viitasalo \& Komi, 1977) reflecting activity levels closer to pre-fatigue EMG values, when compared to placebo and control groups. For CMJ and HopD, we hypothesized that the KT group would have quicker recovery of performance (jump height and hop distance) at 48 hours and 72 hours compared to placebo and control. This information can help healthcare providers by allowing them to more accurately administer KT to patients, and achieve more effective recovery from the symptoms associated with DOMS.

\section{Methods}

\subsection{Participants}

A total of 58 healthy college-age individuals participated in this study. Participants were recruited from one university. Participants had no history of lower extremity injuries in the last 6 months, and engaged in at least four hours of recreational activities per week. In order to obtain the most accurate creatine kinase (CK) levels and effects of DOMS from the eccentric exercise, participants were asked to refrain from any strenuous physical activity 24 hours prior to the first session, until the end of the last data collection session. In addition, the participants were encouraged to stay well hydrated throughout the testing period. The first session consisted of baseline measurements as well as immediately post-exercise measurements. Subjects signed informed consent approved by the Institutional Review Board prior to the beginning of the study.

\subsection{Instrumentation}

2.2.1 Delsys Trigno wireless surface electromyography (EMG) system (Delsys Inc., Boston, MA) was utilized to record muscle activity. Specifications of the Trigno EMG system were as follows: baseline noise < $750 \mathrm{nV}$ RMS, CMRR >80 $\mathrm{dB}$, inter-electrode distance $10 \mathrm{~mm}$, sample rate $2000 \mathrm{~Hz}$, and band-pass filtering of 20-450 Hz ("TRIGNO ${ }^{\mathrm{TM}}$ Wireless System User's Guide ", 2014). EMGWorks software (Delsys Inc.) was utilized to collect the data, and data were analyzed using the custom written code on MatLab (MathWorks, Inc., Natick, MA).

2.2.2 Biodex Isokinetic Dynamometer (Biodex, Shirley, NY) was utilized for the maximum isometric voluntary contractions (MVICs). Biodex was also used during the quadriceps eccentric overload protocol. These protocols were performed in the seated position, with the hip at approximately $90^{\circ}$. The participant's torso and testing limb (thigh and 
lower leg) were secured to the dynamometer with straps (Daneshjoo, Mokhtar, Rahnama, \& Yusof, 2012; Sadeghi et al., 2017).

2.2.3 The capillary blood sample $(300 \mu \mathrm{L})$ from a fingertip was collected for obtaining serum creatine kinase $(\mathrm{CK})$. The capillary blood sample was chosen over the venous sample, because of minimal invasiveness. Studies have shown that the biomarker values from capillary blood samples were comparable to those from venous blood samples (Knoblauch, O'Connor, \& Clarke, 2010; Nunes, Gandra, Alves, Kubota, \& de Macedo, 2006). The Thermo Spectronic 20D+ (Thermo Fisher Scientific, Madison, WI) was utilized to measure serum creatine kinase (CK) levels. Increases in serum CK levels were used as an indirect marker of microtrauma associated with DOMS (Smith et al., 1994).

\subsection{Procedures}

The participants attended a total of three testing sessions over the 72-hour period. The baseline and immediate posteccentric overload measurements were taken on the first session. The participants returned for two more sessions, at 48 hours post-eccentric overload, and at 72 hours post-eccentric overload.

At the beginning of the first session, the capillary blood sample was collected from a fingertip. Participants completed a 5-minute warm-up on a cycle ergometer (Monark 818 E Ergomedic Fitness Bike, Monark Exercise AB, Vansbro, Sweden) at $60 \mathrm{rpm}$ with no resistance, prior to any functional testing. The legs were shaved (if necessary) and cleaned with alcohol pads over the locations of the EMG electrodes attachment. The electrodes were placed on rectus femoris, vastus medialis and vastus lateralis of the testing (dominant) leg (Figure 1). For rectus femoris (RF), the electrode was applied at the half way between the superior pole of the patella and the anterior superior iliac spine (ASIS) (Hermens et al., 1999). For vastus medialis (VM), the electrode was applied at $4 \mathrm{~cm}$ proximal and medial from the medial superior angle of the patella, at the 55-degree angle from the axis of the femur (Cowan, Hodges, Bennel, \& Crossley, 2002). For vastus lateralis (VL), the electrode was applied at the proximal $1 / 3$ of the distance between the ASIS and the superior pole of the patella, $5 \mathrm{~cm}$ away from the line, and at the 15-degree angle from the axis of the femur (Cowan et al., 2002). The EMG electrodes were applied by a single researcher throughout the study to maintain the consistency of the electrode locations.

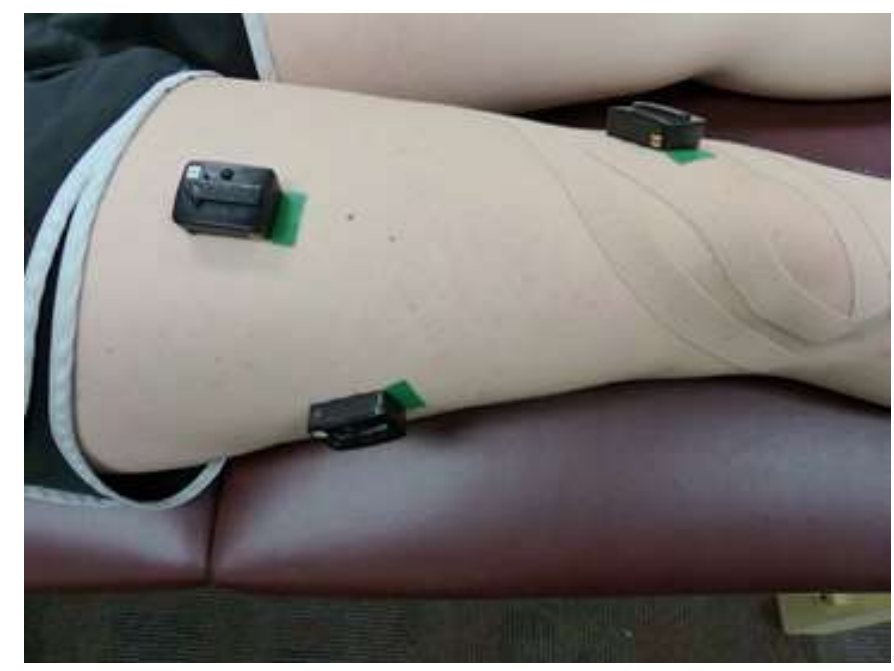

Figure 1. Electrode locations

The subject performed 3 repetitions of 5-second Maximal Voluntary Isometric Contractions (MVIC) of the quadriceps group on the Biodex Isokinetic Dynamometer (Pincivero, Green, Mark, \& Campy, 2000). The participant was placed in the seated position and $45^{\circ}$ knee flexion for the MVICs. Then, two functional activities were performed: countermovement jump (CMJ) and triple single-leg hop for distance (HopD). The CMJ was performed by quickly squatting down followed by a maximal effort jump. The average jump height $(\mathrm{cm})$ from three trials was recorded using the Just Jump mat (Probotics, Inc., Huntsville, AL) (Leard et al., 2007). The HopD was performed by hopping three times in a row as far as possible, while jumping off and landing on the testing leg and sticking the landing at the end. The average hop distance (cm) was recorded from three trials (J. M. Drouin \& Riemann, 2004). The order of functional tests (CMJ and HopD) was randomized.

The participants then underwent 30 maximal eccentric contractions of the quadriceps muscle group on the testing leg, using the Biodex. The exercise was performed from full knee extension to $90^{\circ}$ knee flexion, at $60^{\circ} / \mathrm{sec}$, with an intention of inducing eccentric overload that would result in DOMS. The participant was verbally encouraged to maintain maximal effort throughout the exercise. It has been demonstrated that the measures of mechanical muscle function provide the most effective means of evaluating the magnitude and time course of damage induced by eccentric muscle action (Molina \& Denadai, 2012). Immediately after the eccentric exercise, the participants performed CMJ and HopD, and blood sample was collected for the measurements immediately post exercise.

After the completion of the immediately post-exercise measurements, participants were randomized into one of the three groups: KT, placebo and control. The simple randomization method was used through an online randomization 
number generator program (www.randomizer.org). The investigators were not blinded to the participants' group assignment. Although the participant were aware if they were assigned to a taping or a control group, they were blinded as to the intended therapeutic effects of either taping procedure (KT or placebo). For the KT group, the 7.5-cm wide kinesiology tape (NiTreat Kinesiology Tape, Nitto Medical Corporation, Tokyo, Japan) was applied from the medial mid-thigh to the tibial tuberosity with tape-off tension while the participant lied in the supine position with the knee of the taping leg (dominant leg) in maximal flexion, with the intention of enhancing lymph flow by creating the "pouch" underneath the tape. For the placebo group, the 7.5-cm wide non-woven retention tape (Omnifix Retention Tape, Hartmann-Conco Inc., Rock Hill, SC) was applied on the same location. We selected the different material of tape for the placebo condition, to assess the efficacy of KT because of its stretchy material characteristics. Each taping technique is presented in Figure 2. For all participants, tape remained on the skin for the duration of data collection.

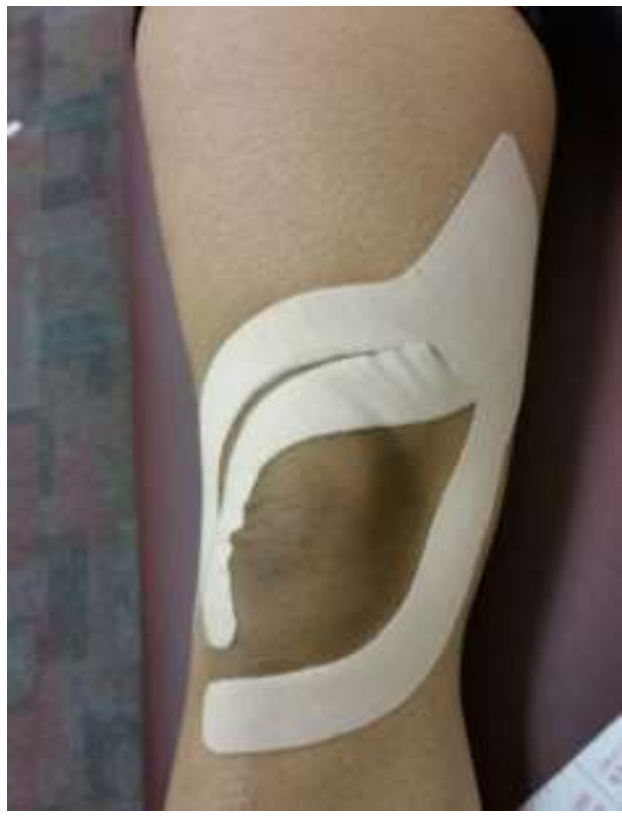

A. Kinesiology tape application

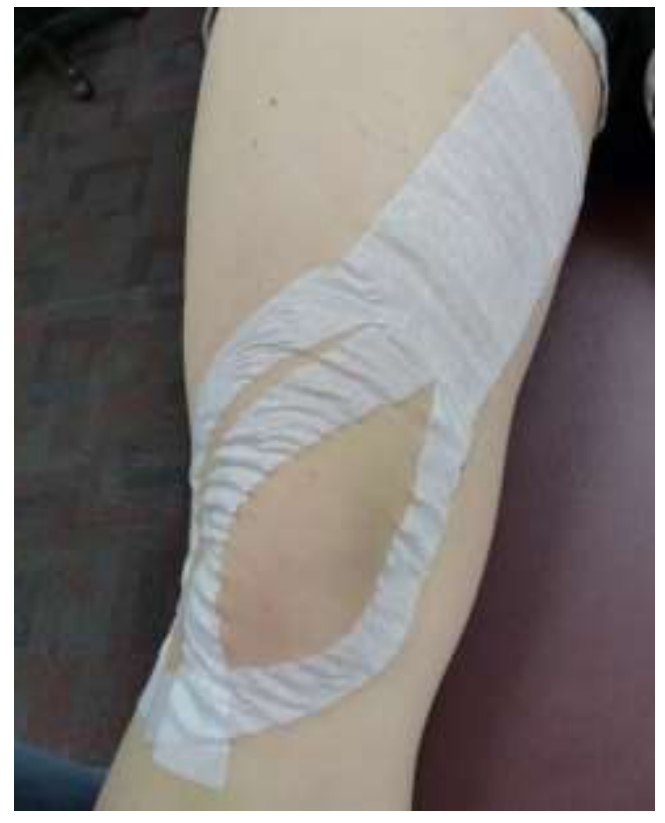

B. Placebo tape application

Figure 2. Taping conditions

The participants were asked to return for second (48 hours post exercise) and third (72 hours post exercise) sessions, at the proposed peaks of DOMS. During these follow-up sessions, the capillary blood sample, jump height/distance and muscle activation during CMJ and HopD were collected.

\subsection{EMG Normalization}

The mean amplitude from the middle 3 seconds of each MVIC trial was used to calculate the average amplitude across the five trials, which served as a reference for normalizing EMG amplitudes during two functional activities (Pincivero et al., 2000). The average EMG amplitude was recorded from three trials of the CMJ and HopD. The EMG activities of vastus medialis (VM), rectus femoris (VM) and vastus lateralis (VL) from each day were normalized to the baseline MVIC activity of each muscle. There are various methods of normalization and advantages and disadvantages associated with each normalization method. We determined that normalization using the baseline (pre-exercise and preDOMS) level of muscle function was the most appropriate for comparing the EMG activities across different time periods as the muscles recover from eccentric overload and subsequent DOMS. The electrodes were placed using the aforementioned guidelines by a single researcher, and traced by a permanent marker, in order to eliminate any potential variability in electrode placement.

\subsection{Statistical Analyses}

Independent variables were Groups (KT, Placebo, Control) and Time (Baseline, Immediately post-exercise, 48 hours, 72 hours). Dependent variables included serum CK level, normalized mean EMG amplitude of each muscle (VM, RF, VL) during CMJ and HopD, CMJ jump height, and HopD distance. For each dependent variable, a separate 3 X 4 repeated measures analysis of variance (ANOVA) was used for statistical analysis (SPSS Version 22, IBM Corp., Armonk, NY). The level of significance was set a priori at $\mathrm{P}<0.05$. In the event of statistically significant results, posthoc pairwise comparisons were made for further identifying the significant differences.

\section{Results}

\subsection{Serum CK Level}

A total of 58 participated in the study. The participants' demographics are shown in Table 1. The results of the serum CK levels across time and groups are presented in Table 2. The main effect of Time on the serum CK level was not statistically significant $\left(\mathrm{F}_{3,53}=2.120, \mathrm{P}=0.131\right)$. Likewise, the main effect of Group on the serum $\mathrm{CK}$ level was not 
statistically significant $\left(\mathrm{F}_{2,55}=0.126, \mathrm{P}=0.882\right)$. Furthermore, the Time-by-Group interaction effect on the serum $\mathrm{CK}$ level was not statistically significant $\left(\mathrm{F}_{6,108}=0.881, \mathrm{P}=0.472\right)$.

Table 1. Participants demographics

\begin{tabular}{cccc}
\hline Variables & KT Group & Placebo Group & Control Group \\
\hline $\mathrm{N}$ & $15($ male=6, female=9) & 19 (male=9, female=10) & 24 (male=11, female=13) \\
\hline Age (years) & $20.47 \pm 1.41$ & $20.16 \pm 1.42$ & $20.71 \pm 1.65$ \\
\hline Height $(\mathrm{cm})$ & $173.10 \pm 8.79$ & $171.98 \pm 8.67$ & $172.80 \pm 9.56$ \\
\hline Mass $(\mathrm{kg})$ & $69.10 \pm 12.35$ & $71.37 \pm 10.21$ & $70.84 \pm 11.54$ \\
\hline $\mathrm{KT}=$ Kinesiology Tape group. Ages, height and mass are presented as mean \pm standard deviation
\end{tabular}

Table 2. Serum creatine kinase and performance results

\begin{tabular}{|c|c|c|c|c|c|c|c|c|c|}
\hline \multicolumn{6}{|c|}{ CK (U/L) } & \multicolumn{2}{|c|}{ HopD (cm) } & \multicolumn{2}{|c|}{$\mathrm{CMJ}(\mathrm{cm})$} \\
\hline & & Mean & SD & & & Mean & SD & Mean & $\mathrm{SD}$ \\
\hline \multirow{3}{*}{ Baseline } & Control & 104.20 & 77.84 & \multirow{3}{*}{ Baseline } & Control & 144.87 & 41.50 & 49.82 & 12.91 \\
\hline & Placebo & 93.31 & 49.33 & & Placebo & 159.1 & 41.57 & 51.57 & 11.25 \\
\hline & KT & 122.41 & 99.21 & & KT & 148.35 & 35.99 & 46.15 & 10.90 \\
\hline \multirow{3}{*}{ Immediate } & Control & 108.48 & 68.74 & \multirow{3}{*}{ Immediate } & Control & 141.72 & 43.21 & 47.48 & 11.07 \\
\hline & Placebo & 92.80 & 63.78 & & Placebo & 150.77 & 42.59 & 49.94 & 12.24 \\
\hline & KT & 141.54 & 117.03 & & KT & 144.08 & 35.74 & 45.93 & 11.56 \\
\hline \multirow{3}{*}{$48 \mathrm{hr}$} & Control & 153.93 & 116.26 & \multirow{3}{*}{$48 \mathrm{hr}$} & Control & 148.7 & 40.99 & 48.57 & 13.45 \\
\hline & Placebo & 141.86 & 172.83 & & Placebo & 157.48 & 42.82 & 49.77 & 10.85 \\
\hline & KT & 119.75 & 57.49 & & KT & 150.78 & 37.08 & 45.82 & 9.53 \\
\hline \multirow{3}{*}{$72 \mathrm{hr}$} & Control & 142.59 & 75.52 & \multirow{3}{*}{$72 \mathrm{hr}$} & Control & 150.19 & 40.52 & 48.19 & 12.15 \\
\hline & Placebo & 133.87 & 120.37 & & Placebo & 161.63 & 42.31 & 50.10 & 9.71 \\
\hline & KT & 127.90 & 74.45 & & KT & 154.88 & 36.39 & 42.89 & 15.86 \\
\hline
\end{tabular}

$\mathrm{CK}=$ serum creatine kinase; HopD = triple single-leg hop for distance; $\mathrm{CMJ}=$ countermovement jump; $\mathrm{KT}=$ Kinesiology Tape group; SD = standard deviation.

\subsection{HopD Performance and EMG Activity}

3.2.1 Table 2 above shows the HopD performance values across groups and time. For HopD distance, a significant main effect of Time $\left(\mathrm{F}_{3,53}=10.562, \mathrm{P}<0.001\right)$ was observed. Post-hoc pairwise comparisons indicated that HopD significantly reduced immediately after the fatigue protocol, and improved at 48 and 72 hours relative to the baseline. There was no significant main effect of Group on HopD $\left(\mathrm{F}_{2,55}=0.403, \mathrm{P}=0.671\right)$. Also, the Time $\mathrm{x}$ Group interaction effect on HopD was not significant $\left(\mathrm{F}_{6,108}=0.592, \mathrm{P}=0.736\right)$.

3.2.2 The results of the EMG activity from VM, RF, and VL during HopD are presented in Table 3. The main effect of Time on VM activity during HopD was significant $\left(\mathrm{F}_{3,53}=6.469, \mathrm{P}=0.002\right)$. However, no significant Group main effect $\left(F_{2,55}=0.619, P=0.543\right)$ or Time $x$ Group interaction $\left(F_{6,108}=0.785, P=0.550\right)$ was found. For $R F$, there was no significant main effect of Time $\left(\mathrm{F}_{3,53}=1.881, \mathrm{P}=0.152\right)$ or Group $\left(\mathrm{F}_{2,55}=0.027, \mathrm{P}=0.974\right)$. Likewise, there was no significant Time $\mathrm{x}$ Group $\left(\mathrm{F}_{6,108}=1.376, \mathrm{P}=0.244\right)$ interaction. The main effect of Time on VL activity during HopD was significant $\left(\mathrm{F}_{3,53}=5.823, \mathrm{P}=0.006\right)$. However, no significant Group main effect $\left(\mathrm{F}_{2,55}=0.204, \mathrm{P}=0.816\right)$ or Time $\mathrm{x}$ Group interaction $\left(\mathrm{F}_{6,108}=0.460, \mathrm{P}=0.739\right)$ was found. 
Table 3. Electromyography (EMG) results of the quadriceps muscles during triple single-leg hop for distance (HopD)

\begin{tabular}{cccccccc}
\hline & \multicolumn{7}{c}{ HopD EMG (\%MVIC) } \\
\hline \multirow{4}{*}{ Baseline } & \multicolumn{2}{c}{ VM } & \multicolumn{2}{c}{ RF } & \multicolumn{2}{c}{ VL } \\
\hline & Control & 159.91 & 55.31 & 110.48 & 59.71 & 172.83 & 76.25 \\
\cline { 2 - 8 } & Placebo & 196.97 & 98.29 & 118.31 & 41.76 & 176.67 & 82.71 \\
\cline { 2 - 8 } Immediate & KT & 191.87 & 65.06 & 93.05 & 22.74 & 153.59 & 40.78 \\
\cline { 2 - 8 } & Control & 159.64 & 67.74 & 110.20 & 66.68 & 183.75 & 112.54 \\
\cline { 2 - 8 } & Placebo & 158.83 & 42.16 & 101.93 & 27.33 & 164.90 & 65.65 \\
\cline { 2 - 8 } & KT & 172.88 & 64.53 & 109.42 & 50.51 & 157.97 & 33.63 \\
\hline \multirow{3}{*}{$48 \mathrm{hr}$} & Control & 185.68 & 117.50 & 134.22 & 120.02 & 154.34 & 72.50 \\
\cline { 2 - 8 } & Placebo & 147.44 & 47.55 & 115.12 & 59.55 & 141.58 & 35.84 \\
\cline { 2 - 8 } & KT & 146.59 & 24.84 & 90.80 & 23.56 & 138.56 & 28.48 \\
\hline \multirow{3}{*}{$72 \mathrm{hr}$} & Control & 150.73 & 65.20 & 94.10 & 38.95 & 132.70 & 39.57 \\
\cline { 2 - 8 } & Placebo & 167.00 & 91.58 & 90.33 & 20.14 & 139.55 & 30.40 \\
\cline { 2 - 8 } & KT & 153.15 & 60.08 & 95.20 & 32.63 & 142.03 & 41.14
\end{tabular}

$\mathrm{VM}=$ vastus medialis; $\mathrm{RF}=$ rectus femoris; $\mathrm{VL}=$ vastus lateralis; $\mathrm{KT}=$ Kinesiology Tape group; $\mathrm{SD}=$ standard deviation.

\subsection{CMJ Performance and EMG Activity}

3.3.1 The CMJ performance values across groups and time are shown in Table 2 above. There was no significant main effect of Time $\left(\mathrm{F}_{3,53}=2.559, \mathrm{P}=0.096\right)$ or Group $\left(\mathrm{F}_{2,55}=0.828, \mathrm{P}=0.442\right)$ on $\mathrm{CMJ}$. Also, the interaction between Time and Group on $\mathrm{CMJ}\left(\mathrm{F}_{6,108}=0.856, \mathrm{P}=0.472\right)$ was not significant.

3.3.2 Table 4 shows the EMG activity of VM, RF and VL during CMJ. The main effect of Time was significant $\left(\mathrm{F}_{3,53}=3.567, \mathrm{P}=0.027\right)$. However, no significant Group main effect $\left(\mathrm{F}_{2,55}=0.323, \mathrm{P}=0.726\right)$ or Time $\mathrm{x}$ Group interaction $\left(\mathrm{F}_{6,108}=1.768, \mathrm{P}=0.134\right)$ was found. For the $\mathrm{RF}$ activity during $\mathrm{CMJ}$, no significant main effect of Time $\left(\mathrm{F}_{3,53}=1.462\right.$, $\mathrm{P}=0.238)$, Group $\left(\mathrm{F}_{2,55}=0.263, \mathrm{P}=0.770\right)$, or Time $\mathrm{x}$ Group interaction $\left(\mathrm{F}_{6,108}=0.825, \mathrm{P}=0.504\right)$ was found. For the $\mathrm{VL}$ activity during CMJ, a significant main effect of Time $\left(\mathrm{F}_{3,53}=5.138, \mathrm{P}=0.008\right)$ was found. No significant main effect of Group $\left(\mathrm{F}_{2,55}=0.126, \mathrm{P}=0.882\right)$ or Time $x$ Group interaction $\left(\mathrm{F}_{6,108}=0.729, \mathrm{P}=0.571\right)$ was found.

Table 4. Electromyography (EMG) results of the quadriceps muscles during countermovement jump (CMJ)

\begin{tabular}{|c|c|c|c|c|c|c|c|}
\hline & \multicolumn{7}{|c|}{ CMJ EMG (\%MVIC) } \\
\hline & & \multicolumn{2}{|c|}{ VM } & \multicolumn{2}{|c|}{$\mathrm{RF}$} & \multicolumn{2}{|c|}{ VL } \\
\hline & & Mean & SD & Mean & SD & Mean & SD \\
\hline \multirow{3}{*}{ Baseline } & Control & 157.93 & 62.60 & 127.92 & 57.13 & 180.99 & 109.79 \\
\hline & Placebo & 166.27 & 54.24 & 135.01 & 37.73 & 186.53 & 105.41 \\
\hline & KT & 188.43 & 70.27 & 122.11 & 27.30 & 157.49 & 45.35 \\
\hline \multirow{3}{*}{ Immediate } & Control & 147.70 & 58.40 & 118.73 & 52.30 & 175.14 & 123.82 \\
\hline & Placebo & 154.23 & 68.99 & 124.79 & 54.04 & 164.33 & 93.72 \\
\hline & KT & 158.29 & 43.09 & 103.89 & 16.82 & 148.19 & 34.52 \\
\hline \multirow{3}{*}{48} & Control & 142.87 & 47.81 & 124.35 & 81.06 & 141.83 & 54.50 \\
\hline & Placebo & 147.89 & 36.05 & 110.21 & 23.05 & 137.78 & 24.60 \\
\hline & KT & 142.39 & 28.31 & 115.45 & 33.34 & 131.47 & 23.85 \\
\hline \multirow{3}{*}{72} & Control & 140.39 & 42.07 & 108.57 & 22.46 & 131.87 & 29.07 \\
\hline & Placebo & 132.52 & 36.96 & 98.20 & 22.48 & 119.62 & 17.40 \\
\hline & KT & 154.58 & 47.96 & 126.35 & 52.00 & 135.19 & 30.09 \\
\hline
\end{tabular}

$\mathrm{VM}=$ vastus medialis; $\mathrm{RF}=$ rectus femoris; $\mathrm{VL}=$ vastus lateralis; $\mathrm{KT}=$ Kinesiology Tape group; $\mathrm{SD}=\mathrm{standard}$ deviation. 


\section{Discussion}

The purpose of this study was to investigate the effects of KT on the serum CK level, and quadriceps muscle activity and functional performance during recovery from DOMS over the 72-hour period of time. Contrary to our hypotheses, our results indicated that the taping conditions (KT, placebo, or control) had no effects on the serum CK level, or quadriceps EMG activity and performance (jump height and distance) during CMJ and HopD, at any time point across the 72-hour period.

This is one of the few studies which has investigated the effects of KT on the serum CK level as the biomarker of muscle tissue damage after eccentric overload. The elevated CK levels may indicate the increased muscle tissue damage and edema as the result of eccentric overload and DOMS (Cheung et al., 2003; Villeco, 2012). Kinesiology Tape is intended to allow or improve blood and lymph flow for edema reduction (J. L. Drouin et al., 2013; Kase et al., 2003). Therefore, we expected that the application of KT would achieve the less increase in the serum CK levels during recovery from DOMS, as compared with the other taping conditions (Placebo and Control). We did not see any effects of KT on the serum CK levels, and our observations were similar to the study performed by Hazar et al (2014). Although DOMS is considered to simulate the injurious condition, the participants in this study were otherwise healthy and injury-free. Previous studies have shown the benefits of KT on swelling and edema reduction in the injured or pathological population (Ristow et al., 2014; Tsai et al., 2009; Villeco, 2012), however the effects of KT on healthy individuals may not be as evident. The large standard deviations could also contribute to the lack of statistically significant findings. The serum CK levels can fluctuate due various factors including physical activities (Baird et al., 2012; Schneider, Dennehy, Rodearmel, \& Hayward, 1995). Our participants were asked to refrain from any strenuous physical activity 24 hours prior to the first session until the end of the 72-hour data collection session. However, completely controlling their activity level was impossible. Since the possibility of individual differences on the baseline CK level may be the reason for not observing the statistically significant differences, we have also conducted a post-hoc ANOVA using the change scores of the serum CK level at different time points relative to the baseline (Immediate Baseline, 48-hour - Baseline, 72-hour - Baseline). We did not observe any statistically significant differences, and therefore we conclude that the serum CK level during the recovery from DOMS was not largely affected by the application of KT. Other therapeutic modalities such as soft tissue massage have achieved reduction in CK level, pain and swelling (Zainuddin, Newton, Sacco, \& Nosaka, 2005), and therefore modalities other than KT may be more effective in improving blood/lymph flow and reducing local edema after DOMS.

The lack of statistically significant effects of $\mathrm{KT}$ on quadriceps muscle activity and functional performance is contradictory to our initial hypothesis. KT is proposed to enhance muscle functions (Kase et al., 2003), possibly due to the increased cutaneous input for enhancing the neuromuscular functions. The muscle function improvements have been seen more in those with injuries or pathology such as shoulder impingement syndrome (Hsu, Chen, Lin, Wang, \& Shih, 2009) and patellofemoral pain (P. L. Chen, Hong, Lin, \& Chen, 2008). On the contrary, KT seems to have minimal to no effects on muscle functions and performance, regardless of the duration of tape application, in the healthy population (Briem et al., 2011; J. L. Drouin et al., 2013; Fu et al., 2008; Janwantanakul \& Gaogasigam, 2005; Poon et al., 2015). A study comparable to this current study, conducted by Ozmen et al, observed that KT maintained muscle flexibility for 2 days after DOMS, but had no effects on performance (Ozmen et al., 2016). Single leg hops and countermovement jumps are whole-body exercises that demand contributions from various muscles, however could require significant contributions from the quadriceps muscle group (Cowan et al., 2002; Orishimo \& Kremenic, 2006; Stensdotter, Hodges, Mellor, Sunderlin, \& Hager-Ross, 2003). Our data illustrates that quadriceps activity stays diminished compared to the baseline, even at 72 hours post-eccentric exercises; however, different taping conditions did not improve or hinder quadriceps activity. Also, despite slightly diminished activity of quadriceps across time, our results indicated that neither taping conditions nor time yielded any differences on CMJ vertical height or HopD distance. These findings suggest the possibility of recruitment of other muscles of the lower extremity contributing to maintain the functional performance. It is out of the scope of this study to investigate the effects of DOMS on compensatory biomechanical alterations during CMJ and HopD. However, we can conclude that KT does not influence serum CK level, muscular activity and functional performance while recovering from DOMS.

The unequal sample size in each group may be a limitation of the study. This variation occurred due to a few participants dropping out of the study. A larger sample size would be helpful, however, other comparable studies have similar sample sizes. Inclusion of both genders could be another limitation, since males tend to have higher baseline serum CK levels compared to females (Baird et al., 2012). The effects of gender on recovery from DOMS are not very obvious (Dannecker et al., 2012; Nie, Kawczynski, Madeleine, \& Arendt-Nielsen, 2005). Future studies may investigate the combination of gender and KT on recovery from DOMS. The standardized eccentric overload protocol of 30 maximum-effort eccentric knee flexion was selected to induce DOMS on the quadriceps muscle group. This protocol has been established as an appropriate protocol for inducing DOMS (T. C. Chen, Lin, Chen, Lin, \& Nosaka, 2011), through the pilot testing prior to the data collection phase. However, any standardized exercise protocol could be a limitation in that the extent of the subsequent DOMS or muscle damage may not be the same across all participants. A future study may compare the effects of KT on muscle function and recovery from two different eccentric overload protocols (individualized vs. standardized).

\section{Conclusion}

This was the first study to our knowledge that investigated the effects of KT on serum CK levels, along with the quadriceps EMG activity and functional performance of CMJ and HopD, during recovery from DOMS. Our results did 
not demonstrate the effects of KT on any of the outcome variables. While no negative or harmful effects of KT were seen from our study, the healthcare providers must use caution when selecting the most appropriate treatment modalities for those experiencing DOMS.

\section{References}

Baird, M. F., Graham, S. M., Baker, J. S., \& Bickerstaff, G. F. (2012). Creatine-kinase- and exercise-related muscle damage implications for muscle performance and recovery. Journal of Nutrition and Metabolism, 2012, 13. doi: $10.1155 / 2012 / 960363$

Bassett, K. T., Lingman, S. A., \& Ellis, R. F. (2010). The use and treatment efficacy of kinesthetic taping for musculoskeletal conditions: a systematic review. New Zealand Journal of Physiotherapy, 38(2), 56-62.

Briem, K., Eythorsdottir, H., Magnusdottir, R. G., Palmarsson, R., Runarsdottir, T., \& Sveinsson, T. (2011). Effects of kinesio tape compared with nonelastic sports tape and the untaped ankle during a sudden inversion perturbation in male athletes. Journal of Orthopaedic and Sports Physical Therapy, 41(5), 328-335. doi: 10.2519/jospt.2011.3501

Chen, P. L., Hong, W. H., Lin, C. H., \& Chen, W. C. (2008). Biomechanics effects of Kinesio taping for persons with patellofemoral pain syndrome during stair climbing. Paper presented at the 4th Kuala Lumpur International Conference on Biomechanical Engineering.

Chen, T. C., Lin, K. Y., Chen, H. L., Lin, M. J., \& Nosaka, K. (2011). Comparison in eccentric exercise-induced muscle damage among four limb muscles. European Journal of Applied Physiology, 111, 211-223.

Cheung, K., Hume, P. A., \& Maxwell, L. (2003). Delayed onset muscle soreness treatment strategies and performance factors. Sports Medicine, 33(2), 145-164.

Cowan, S. M., Hodges, P. W., Bennel, K. L., \& Crossley, K. M. (2002). Altered vastii recruitment when people with patellofemoral pain syndrome complete a postural task. Archives of Physical Medicine and Rehabilitation, 83, 989-995. Curtis, D., Fallows, S., Morris, M., \& McMakin, C. (2010). The efficacy of frequency specific microcurrent therapy on delayed onset muscle soreness. Journal of Bodywork and Movement Therapy, 14, 272-279.

Daneshjoo, A., Mokhtar, A. H., Rahnama, N., \& Yusof, A. (2012). The effects of injury preventive warm-up programs on knee strength ratio in young male professional soccer players. PLoS ONE, 7(12), e50979. doi: 10.1371/journal.pone.0050979

Dannecker, E. A., Liu, Y., Rector, R. S., Thomas, T. R., Fillingim, R. B., \& Robinson, M. E. (2012). Sex differences in exercise-induced muscle pain and muscle damage. Journal of Pain, 13(12), 1242-1249. doi: 10.1016/j.jpain.2012.09.014.

Drouin, J. L., McAlpine, C. T., Primak, K. A., \& Kissel, J. (2013). The effects of kinesiotape on athletic-based performance outcomes in healthy, active individuals: a literature synthesis. Journal of Canadian Chiropractic Association, 57(4), 356-365.

Drouin, J. M., \& Riemann, B. L. (2004). Lower extremity functional performance testing, part 1. Athletic Therapy Today, 9(2), 46-49.

Duruturk, N., Pekyavas, N., Yilmaz, A., \& Karatas, M. (2016). The effects of quadriceps kinesio taping on aerobic and anaerobic exercise performance in healthy participants: a randomized controlled study. International Journal of Athletic Therapy and Training, 21(2), 32-38. doi: http://dx.doi.org/10.1123/ijatt.2014-0137

Fu, T.-C., Wong, A. M. K., Pei, Y.-C., Wu, K. P., Chou, S.-W., \& Lin, Y.-C. (2008). Effect of Kinesio taping on muscle strength in athletes - A pilot study. Journal of Science and Medicine in Sport, 11, 198-201.

Hermens, H. J., Merletti, F. R., Hagg, G. G., Stegeman, D., Blok, J., Rau, G., \& Disselhorst-Klug, C. (1999). SENIAM 8. European recommendations for sufrace electromyography, deliverable of the SENIAM project: Roessingh Research and Development.

Howell, J. M., Chleboum, G. S., \& Conaster, R. R. (1993). Muscle stiffness, strength loss, swelling and soreness following exercise induced injury to humans. Journal of Physiology, 464, 183-196.

Hsu, Y.-H., Chen, W.-Y., Lin, H.-C., Wang, W. T. J., \& Shih, Y.-F. (2009). The effects of taping on scapular kinematics and muscle performance in baseball players with shoulder impingement syndrome. Journal of Electromyography and Kinesiology, 19, 1092-1099.

Janwantanakul, P., \& Gaogasigam, C. (2005). Vastus lateralis and vastus medialis obliquus muscle activity during the application of inhibition and facilitation taping techniques. Clinical Rehabilitation, 19(1), 12-19.

Kase, K., Wallis, J., \& Kase, T. (2003). Clinical therapeutic applications of the kinesio taping method (2nd Ed ed.). Tokyo, Japan: Ken Ikai Co. Ltd.

Knoblauch, M. A., O'Connor, D. P., \& Clarke, M. S. F. (2010). Capillary and venous samples of total creatine kinase are similar after eccentric exercise. Journal of Strength and Conditioning Research, 24(12), 3471-3475.

Leard, J. S., Cirillo, M. A., Katsnelson, E., Kimiatek, D. A., Miller, T. W., Trebincevic, K., \& Garbalosa, J. C. (2007). Validity of two alternative systems for measuring vertical jump height. Journal of Strength and Conditioning Research, 21(4), 1296-1299.

McHugh, M. P., Connolly, D. A., Eston, R. G., \& Gleim, G. W. (1999). Exercise-induced muscle damage and potential mechanisms for the repeated bout effect. Journal of Sports Medicine, 27, 158-170.

Molina, R., \& Denadai, B. S. (2012). Dissociated time course recovery between rate of force development and peak torque after eccentric exercise. Clinical Physiology and Functional Imaging, 32, 179-184. doi: 10.1111/j.1475097X.2011.01074.X

Nie, H., Kawczynski, A., Madeleine, P., \& Arendt-Nielsen, L. (2005). Delayed onset muscle soress in neck/shoulder muscles. European Journal of Pain, 9, 653-660. doi: 10.1016/j.ejpain.2004.12.009 
Nunes, L. A. S., Gandra, P. G., Alves, A. A., Kubota, L. T., \& de Macedo, D. V. (2006). Adequacies of skin puncture for evaluating biochemical and hematological blood parameters in athletes. Clinical Journal of Sports Medicine, 16(5), 418-421.

Orishimo, K. F., \& Kremenic, I. J. (2006). Effect of fatigue on single-leg hop landing biomechanics. Journal of Applied Biomechanics, 22, 245-254.

Ozmen, T., Aydogmus, M., Dogan, H., Acar, D., Zoroglu, T., \& Willems, M. (2016). The effect of Kinesio Taping on muscle pain, spring performance, and flexibility in recovery from squat exercise in young adult women. Journal of Sport Rehabilitation, 25, 7-12.

Page, P. (1995). Pathophysiology of acute exercise-induced muscular injury: clinical implications. Journal of Athletic Trainining, 30(1), 29-34.

Pincivero, D. M., Green, R. C., Mark, J. D., \& Campy, R. M. (2000). Gender and muscle differences in EMG amplitude and median frequency, and variability during maximal voluntary contractions of the quadriceps femoris. Journal of Electromyography and Kinesiology, 10, 189-196.

Poon, K. Y., Li, S. M., Roper, M. G., Wong, M. K. M., Wong, O., \& Cheung, R. T. H. (2015). Kinesiology tape does not facilitate muscle performance: a deceptive controlled trial. Manual Therapy, 20, 130-133.

Prasartwuth, O., Taylor, J. L., \& Gandevia, S. C. (2005). Maximal force, voluntary activation and muscle soreness after eccentric damage to human elbow flexor muscles. Journal of Physiology, 567(1), 337-348.

Ristow, O., Pautke, C., Kehl, V., Koerdt, S., Schwarzler, K., Hahnefeld, L., \& Hohlweg-Majert, B. (2014). Influence of kinesiologic tape on postoperative swelling, pain and trismus after zygomatico-orbital fractures. Journal of CranioMaxillo-Facial Surgery, 42(469-476).

Sadeghi, H., Hakim, M. N., Hamid, T. A., Amri, S. B., Raxeghi, M., Frarazdaghi, M., \& Shakoor, E. (2017). The effect of exergaming on knee proprioception in older men: a randomized controlled trial. Archives of Gerontology and Geriatrics, 69, 144-150. doi: 10.1016/j.archger.2016.11.009

Schneider, C. M., Dennehy, C. A., Rodearmel, S. J., \& Hayward, J. R. (1995). Effects of physical activity on creatine phosphokinase and the isoenzyme creatine kinase-MB. Annals of Emergency Medicine, 25(4), 520-524.

Slupik, A., Dwornik, M., Bialoszewski, D., \& Zych, E. (2007). Effect of Kinesio Taping on bioelectrical activity of vastus medialis muscle. Preliminary report. Ortopedia Traumatologia Rehabilitacja, 9(6), 644-651.

Smith, L. L., Fulmer, M. G., Holbert, D., McCammon, M. R., Houmard, J. A., Frazer, D. D., . . Israel, R. G. (1994). The impact of a repeated bout of eccentric exercise on muscular strength, muscle soreness and creatine kinase. British Journal of Sports Medicine, 28(4), 267-271.

Stensdotter, A. K., Hodges, P. W., Mellor, R., Sunderlin, G., \& Hager-Ross, C. (2003). Quadriceps activation in closed and in open kinetic chain exercise. Medicine and Science in Sports and Exercise, 35(12), 2043-2047.

Szasz, G., Gruber, W., \& Bernt, E. (1976). Creatine kinase in serum: 1. determination of optimum reaction conditions. Clinical Chemistry, 22(5), 650-656.

Tee, J. C., Bosch, A. N., \& Lambert, M. I. (2007). Metabolic consequences of exercise induced muscle damage. Sports Medicine, 37(10), 827-836.

Thelen, M. D., Dauber, J. A., \& Stoneman, P. D. (2008). The clinical efficacy of Kinesio tape for shoulder pain: a randomized, double-blinded, clinical trial. Journal of Orthopaedic and Sports Physical Therapy, 38(7), 389-395.

TRIGNO $^{\text {TM }}$ Wireless System User's Guide (2014). Retrieved December 28, 2016, from http://delsys.com/Attachments_pdf/manual/MAN-012-2-7.pdf

Tsai, H. J., Hung, H. C., Yang, J. L., Huang, C. S., \& Tsauo, J. Y. (2009). Could kinesio tape replace the bandage in decongestive lymphatic therapy for breast-cancer-related lypmhedema? A pilot study. Supportive Care in Cancer, 17, 1353-1360.

Viitasalo, J. H. T., \& Komi, P. V. (1977). Signal characteristics of EMG during fatigue. European Journal of Applied Physiology, 37(2), 111-121.

Villeco, J. (2012). Edema, a silent but important factor. Journal of Hand Therapy, 25, 153-162.

Wallimann, T., Wyss, M., Brdiczka, D., Nicolay, K., \& Eppenberger, H. M. (1992). Intracellular compartmentation, structure and function of creatine kinase isoenzymes in tissues with high and fluctuating energy demands: the 'phosphocreatine circuit' for cellular energy homeostasis. Biochemical Journal, 281, 21-40.

Williams, S., Whatman, C., Hume, P., \& Sheerin, K. (2012). Kinesio taping in treatment and prevention of sports injuries: a meta-analysis of the evidence of its effectiveness. Sports Medicine, 42(2), 153-164.

Zainuddin, Z., Newton, M., Sacco, P., \& Nosaka, K. (2005). Effects of massage on delayed-onset muscle soreness, swelling, and recovery of muscle function. Journal of Athletic Trainining, 40(3), 174-180. 